\title{
Facebook como herramienta de comunicación en procesos educativos*
}

\author{
Facebook as a Communication Tool in Educative Processes
}

Facebook como ferramenta de comunicação nos processos educativos

Cecilia Molina Loyola ${ }^{\text {a }}$

Universidad de Cuenca, Ecuador

cecilia.molina@ucuenca.edu.ec

ORCID: https://orcid.org/0000-0002-1131-0624

Fernando Ortiz Vizuete

Universidad de Cuenca, Ecuador

ORCID: https://orcid.org/0000-0001-5824-3425

Bolivar Ávila Solano

Universidad de Cuenca, Ecuador

ORCID: https://orcid.org/0000-0003-4840-5085

Ángela Maria Maldonado Vélez

Universidad de Cuenca, Ecuador

ORCID: https://orcid.org/0000-0002-7996-0690
DOI: https://doi.org/10.11144/Javeriana.syp39-76.fhcp

Recibido: 27 Julio 2018

Aceptado: 30 Marzo 2019

Publicado: 30 Junio 2020

\section{Resumen:}

Este artículo se enmarca en el proyecto de investigación "Usos y aplicaciones de la Red Social Facebook en la Universidad de Cuenca". Nos interesó conocer si Facebook ha generado escenarios de aprendizaje complementarios a los procesos formales y escolarizados o si constituye una herramienta de comunicación en las dinámicas educativas. La metodología se basó en una encuesta aplicada a 375 estudiantes y 287 docentes de la Universidad de Cuenca, grupos focales y entrevistas a profundidad realizadas a profesores de diferentes facultades. El artículo analiza a la red social como una herramienta de mediación entre profesores y estudiantes. Se plantea la necesidad de establecer procesos más abiertos para comprender las formas cómo los estudiantes se comunican, aprenden y conviven en ella, al ser un escenario donde interactúan docente-estudiante, estudiante-estudiante y que se vislumbra como un espacio para establecer diálogos orientados a la generación de conocimientos compartidos.

Palabras clave: Facebook, comunicación, educación, redes sociales, universidad.

\section{Abstract:}

This article is conducted under the framework of the research Project "Uses and Applications of Facebook at the University of Cuenca". We are very interested in determining whether Facebook has created learning scenarios to compliment the formal schooling processes or, on the other hand, it is a communication tool in the educative dynamics. The methodology consists in a survey applied to 375 students and 287 teachers at the Universidad de Cuenca, some focus groups and some in-depth interviews to teachers from different departments. This article analyzes this social network as a tool that mediates between teachers and students. It also addresses the need to establish more open processes in order to understand how the students communicate, learn and coexist at college, as it is a scenario where teacher-student and student-teacher interactions occur. It is also deemed as a space to start dialogues intended to gain shared insights.

Keywords: Facebook, communication, education, social networks, university.

\section{Resumo:}

Este artigo faz parte do projeto de pesquisa "Usos e aplicações da Rede Social Facebook na Universidade de Cuenca". Interessounos conhecer se Facebook gerou cenários de aprendizagem complementares aos processos formais e escolarizados ou se constitui uma ferramenta de comunicação nas dinâmicas educacionais. A metodologia baseou-se num inquérito aplicado a 375 alunos e 287 docentes da Universidade de Cuenca, grupos focais e em entrevistas a profundidade com professores de diferentes faculdades. $\mathrm{O}$ artigo analisa a rede social como ferramenta de mediação entre professores e alunos. Coloca-se a necessidade de estabelecer

Notas de autor

\footnotetext{
${ }^{a}$ Autora de correspondencia. Correo electrónico: cecilia.molina@ucuenca.edu.ec
} 
processos mais abertos para compreender as formas como os alunos se comunicam, aprendem e convivem nela, visto que se trata de um cenário onde interagem docente-aluno, aluno-aluno e que se vislumbra como espaço para estabelecer diálogos orientados à geração de conhecimentos compartilhados.

Palavras-chave: Facebook, comunicação, educação, redes sociais, universidade.

\section{Introducción}

La red ha cambiado la vida cotidiana de las personas e insertado nuevos repertorios a las formas de comunicación al igual que estrategias de acción social, así mismo abre horizontes para la creación, generación de conocimientos, participación y colaboración en diferentes ámbitos desde la recreación, política y procesos educativos (Alonso y Alonso, 2014) (Menéndez y Sánchez, 2013).

Facebook en un espacio desterritorializado caracterizado por la inmediatez, tiene a su favor la posibilidad de conformar comunidades virtuales en donde las personas tienen la oportunidad de comunicarse de manera horizontal e intervenir en asuntos de interés social y público. Esta característica de posibilitar diferentes agrupamientos sociales, es un atractivo para los millones de usuarios, es por eso que algunos estudios hablan de esta plataforma como un espacio virtual de convergencia de medios, culturas heterogéneas y audiencias participativas (Sued, 2010). Estos elementos permiten que jóvenes universitarios integren fácilmente esta red social en sus actividades estudiantiles pues al mismo tiempo que realizan su vida social, comparten o se informan sobre los aspectos académicos.

Para los nativos digitales, Facebook es un lugar natural. Los migrantes digitales se adaptan con facilidad a su arquitectura, pues el manejo de Facebook es bastante sencillo (Sued, 2010) Facebook se convierte en un espacio de intercambio y convergencia, un lugar donde se generan tanto transacciones como procesos comunicativos. A partir de diversas investigaciones se concluye que esta plataforma es una poderosa herramienta de alfabetización digital, con potencialidades para configurar comunidades virtuales o para aportar en procesos educativos (Piscitelli, 2009; Alcoceba, 2013; Alonso y Alonso, 2014; Esquivel y Rojas, 2014; Fondevila et al., 2015; Lambic, 2016).

Actualmente los dispositivos tecnológicos y las plataformas digitales, principalmente Facebook, están presentes en el aula, situación que obliga a los docentes a adaptarse a esta realidad para un uso inteligente, responsable, crítico y constructivo (Fondevila et al., 2015). Por otro lado, varios estudios demostraron que Facebook es perfectamente compatible con las actividades académicas proponiendo cuestionamientos a los conceptos y prácticas de enseñanza-aprendizaje. Si bien Facebook no es una plataforma pedagógica, los estudiantes utilizan esta red social para actividades estudiantiles como la conformación comunidades de aprendizaje, generación de conocimiento propio, publicación de audiovisuales e integración de procesos colaborativos (Fondevila et al., 2015). Así mismo se han clasificado algunas aplicaciones educativas de Facebook por ejemplo: constituir un entorno colaborativo a través de la conformación de comunidades virtuales; medio alterno de comunicación entre docentes y estudiantes; escenario propicio para aprendizaje de idiomas; plataforma virtual de aprendizaje; espacio para la generación de mayor participación e interactividad de los estudiantes y para la ejecución de debates en línea sobre los temas planteados en el aula; plataforma usada para la realización de asesoría académica (Esquivel y Rojas, 2014).

Los jóvenes integran a Facebook en sus actividades estudiantiles independientemente de sus docentes para compartir información académica con sus pares, por lo tanto, tiene un valor educativo por las posibilidades de intercambio de información y comunicación, en esta nueva realidad el docente tiene la urgencia de sintonizar con estos nuevos lenguajes juveniles y sus formas de interacción. Las exigencias actuales muestran que el rol del docente está cambiando de controlador hacia un promotor, mediador y gestor de la información (Fondevila et al., 2015; Martínez, 2017). 
Frente a los desafíos de la hiper conectividad de los jóvenes, algunos estudios plantean la necesidad de adaptar el conocimiento académico a los nuevos soportes, entre ellos Facebook pues su diseño se adapta fácilmente a las dinámicas de comunicación en escenarios educativos dado que sigue siendo la red más utilizada y valorada por estudiantes. Algunos estudios señalan que las redes sociales ofrecen un aspecto de interacción y cooperación (Rodríguez et al., 2017). En ámbitos universitarios Facebook es visto como un nuevo escenario de intercambio profesor-estudiante, un complemento de la docencia y como espacio de comunicación, que a diferencia de otras plataformas no esperan al estudiante, sino que es buscada por los jóvenes porque está en su rutina diaria (Tuñez y Sixto, 2012).

Por otro lado, hay quienes ven con preocupación la llegada de las redes sociales porque pueden consolidar una forma de pensamiento superficial fomentando una cultura "ligth", que deviene en la distracción, efímera y en movimiento que solo toca la superficie (Serrano, 2017). Estas reflexiones se han planteado en el ámbito educativo pues algunas investigaciones demuestran que los estudiantes pasan mucho tiempo en las redes sociales en desmedro de sus actividades académicas. Sin embargo, es una realidad con la que hay que convivir, hoy la universidad no puede permanecer ajena a la presencia cada vez más invasiva de las redes sociales en la vida de los estudiantes.

En definitiva, esta red virtual plantea algunos desafíos a la tradición académica para avanzar hacia la conformación de tejidos y dinámicas sociales de aprendizaje compartido. Estas redes podrían considerarse como una apuesta hacia la convergencia de ideas, propuestas e iniciativas con grandes aportes en la dimensión académica. Las redes virtuales como nuevos medios de comunicación ofrecen estas opciones que deberán ser abordadas como estrategias comunicativas integradas a los procesos de enseñanza-aprendizaje (Piscitelli et al., 2010; Menéndez y Sánchez, 2013; Esquivel y Rojas, 2014; Lambic, 2016; Fondevila et al., 2015).

Tanto Internet como las redes sociales, desafían las concepciones tradicionales de los modelos de aprendizaje porque hoy tenemos un sujeto digital que accede a un volumen ilimitado de información que no necesariamente se traduce en conocimiento, al respecto el rol del docente es precisamente orientar y facilitar los procesos de selección de esa información, para convertirla en conocimiento (Esquivel y Rojas, 2014). Así mismo los avances tecnológicos desafían la generación de prácticas educativas con las que se integra la dimensión humana para un aprendizaje dialogante y participativo (Marta-Lazo y Gabelas, 2017).

La esencia del proceso de aprendizaje consiste en apuntar a la formación de seres autónomos, solidarios, con capacidades críticas para transformar sus realidades. Las propuestas de un proceso de aprendizaje transformador en tiempos contemporáneos, nos remite al pensamiento de Paolo Freire (1969) que sigue desafiando aquellos modelos de educación tradicional que se sustentan en la repetición y no en la transformación, para plantear el proceso educativo como un encuentro dialógico, de investigación, análisis y debate, pues como indica que el ser humano es un ser de relaciones, no solamente de contactos, así mismo, no sólo está en el mundo sino con el mundo, en donde establece una pluralidad de relaciones con los otros, pero también con los datos que ofrece la realidad. Este pensamiento crítico que sitúa al ser humano con las potencialidades para estar "con el mundo", le permite crear y recrear, participar y transformar esa realidad, en definitiva, hacer su historia, a partir de la posibilidad de decidir. Esta contribución de Freire (1969) cobra actualidad en esta era virtual en la que emerge un ser digital, que solamente puede constituirse como un sujeto, desde una conciencia crítica, que interpreta ese mundo para transformarlo.

Las redes sociales pueden ofrecer esa posibilidad de un trabajo cooperativo y de la creación de comunidades colaborativas de aprendizaje y pensamiento abierto, si se supera el gregarismo como miedo a la soledad, y se asume el desafío como dice Freire de "comprender el misterio de los cambios, sin ser un juguete de ellos" (1969, p. 37). De ahí que los procesos educativos pueden integrar estos nuevos desafíos que plantean las plataformas digitales y las redes sociales en internet, desde una perspectiva crítica para convertirlas en herramientas de transformación, a partir de una práctica de permanente reflexión y acción. Además, ofrecen la posibilidad de establecer diálogos horizontales entre pares y entre docente-estudiante, diálogos horizontales entre dos sujetos que hablan sobre un tema, desde una visión comprometida, afectiva y crítica. 


\section{Metodología}

Esta investigación emerge en el contexto del proyecto: "Estudio sobre usos y aplicaciones de la red social Facebook en la Universidad de Cuenca", cuyo propósito es estudiar los usos de Facebook en la perspectiva de generar conocimientos que aporten a los procesos de enseñanza aprendizaje y las estrategias comunicativas con base en un saber compartido hacia mejores prácticas interactivas digitales. El proceso de investigación integra varios tópicos como usos de las redes, derechos, libertades, la construcción de la identidad digital, las preferencias con relación a las plataformas digitales y los usos académicos. La investigación está llevada por docentes de la carrera de Comunicación Social de la Universidad de Cuenca.

Sin lugar a dudas, los estudiantes están conectados, buena parte de sus interacciones se realizan desde la virtualidad pues los medios digitales están ahí, seduciendo y planteando nuevas formas de comunicación o interesantes estrategias de expresión y auto representación. En este contexto, nos preguntamos ¿qué nos mueve a estar en Facebook? Varias son las razones para ingresar plenamente en esta red, así mismo son distintos los usos y discursos que en ella se generan. Entre las motivaciones está la construcción de una identidad, la facilidad para establecer relaciones, compartir afectos, conectarse con un gran número de personas, acceder a un importante caudal de información, unirse a grupos de interés, manifestarse públicamente sobre asuntos de corte social, político, ambiental, económico, para generar una presencia pública y salir del anonimato o simplemente para seguir las tendencias de consumo material o simbólico.

El desconocimiento sobre los usos que estudiantes y docentes hacen de Facebook, motivó una investigación amplia y significativa que permite, desde una mirada crítica y sin prejuicios, abordar este interrogante.

Nos interesó conocer si esta red social ha generado escenarios de aprendizaje complementarios a los procesos formales y escolarizados, o, si constituye una herramienta de comunicación para los procesos de enseñanza aprendizaje.

Para el desarrollo de la investigación realizada en el proyecto "Estudio sobre usos y aplicaciones de la red social Facebook en la Universidad de Cuenca”, se integraron métodos cualitativos y cuantitativos. Se aplicó una encuesta tanto a estudiantes como a docentes, se realizaron grupos focales de análisis con docentes así como entrevistas a profundidad a docentes y estudiantes.

Se optó por la ejecución de encuestas a estudiantes y docentes de las diferentes facultades de la Universidad de Cuenca durante el periodo septiembre 2016 a febrero 2017. Al considerar que tanto estudiantes como profesores poseen características distintas, se determinó un diseño muestral para cada conjunto, realizando un muestreo estratificado por afijación proporcional para cada conjunto poblacional. Se consideró un $95 \%$ de confianza y un error muestral del 5\%, el resultado de dicho proceso fue la aplicación de 375 encuestas a estudiantes y 287 a docentes. El procesamiento de la información fue realizado en el programa estadístico SPSS 20.

La encuesta abarcó varios aspectos, entre los que están: usos de las redes sociales, uso de la red social Facebook y actividades académicas. Además, se realizó un seguimiento a los perfiles o páginas de Facebook de la Universidad, las facultades, los departamentos, proyectos de investigación y las asociaciones de estudiantes con el fin de tener un conocimiento sobre su interacción en la red.

\section{Resultados}

Los resultados de la encuesta muestran que un $95 \%$ de estudiantes y un $86 \%$ de docentes tienen una cuenta en Facebook, además en la Universidad de Cuenca tanto sus facultades como los diferentes departamentos y las asociaciones de estudiantes, mantienen al menos 86 páginas y perfiles en esta plataforma.

Los resultados además muestran las preferencias que tienen los estudiantes y docentes hacia otras redes sociales. El 92\% de estudiantes y $86 \%$ de profesores encuestados usan WhatsApp; mientras que un $90 \%$ de 
estudiantes y un $80 \%$ de docentes utilizan YouTube; siendo Facebook, WhatsApp y YouTube las herramientas de internet de mayor uso en la Universidad de Cuenca. Sin dejar de lado, redes sociales como Instagram que tiene gran acogida en los estudiantes, un $84 \%$ de ellos poseen cuenta en esta red social; mientras que solo un $47 \%$ de docentes poseen una cuenta en Instagram. Una herramienta que utiliza un $61 \%$ de docentes de la Universidad de Cuenca es Google Plus, mientras que solo un 51\% de estudiantes utilizan dicha plataforma. Con relación a Twitter los datos muestran una similitud entre estudiantes y docentes en el uso de esta red social con un $54 \%$ para cada sector poblacional, esto evidencia que esta plataforma resulta menos atractiva.

Los resultados evidencian que un $89,86 \%$ de estudiantes y un $66,54 \%$ de profesores se conectan a las redes sociales principalmente mediante un teléfono inteligente. Este dato señala que el teléfono se ha convertido en ese dispositivo de acceso para todos los sectores sociales, como un multimedia portátil porque se puede almacenar fotos, videos, documentos, textos y la posibilidad de establecer una comunicación a través de mensajes o llamadas. Esta característica de la portabilidad está permitiendo que los estudiantes estén conectados todo el tiempo provocando una ruptura de la concepción espacio-tiempo; se vive, se actúa en simultáneo en distintos espacios.

Con relación a las redes sociales y las actividades académicas los resultados dan cuenta de una creciente incorporación de Facebook en actividades relacionadas con los procesos educativos, principalmente los estudiantes utilizan la plataforma de múltiples maneras y adaptan la plataforma a sus necesidades.

Un $77,80 \%$ de estudiantes y un $56,20 \%$ de docentes tienen entre amigos al perfil institucional de la Universidad, lo que permite estar actualizados sobre las actividades de la Universidad y acceder a esta información mientras se navega o se interactúa con las redes de amigos o conocidos. Este comportamiento, mayoritariamente entre los estudiantes, evidencia una continuidad entre la vida social y la profesional. Ya no existen espacios o tiempos diferenciados.

El 61,10\% de estudiantes tiene entre sus amigos a profesores de la Universidad, mientras un 62,70\% de docentes tiene entre sus contactos a estudiantes. Esta realidad nos permite advertir que en Facebook se buscan conexiones en lo académico. Tanto estudiantes como docentes pueden conocer a la otra persona en sus aspectos cotidianos, acercarse a sus comportamientos fuera de la Universidad, descubrir sus aficiones y sus redes de amistades. Ahora docentes y estudiantes se están observando mutuamente lo cual puede aportar con información significativa para comprender "al otro", definitivamente los docentes pueden tener un acercamiento al "mundo" de los estudiantes como un elemento importante en el entendimiento integral de sus realidades, lenguajes y temas de interés.

De las entrevistas realizadas a docentes se desprende que aún hay posiciones divididas con relación a los posibles usos académicos de Facebook, algunos señalan que Facebook es una red para contactar a sus amigos, por tanto, no debería incluirse a los estudiantes, pues para las actividades educativas se disponen de otras plataformas diseñadas con esas finalidades como Moodle. Sin embargo, los estudiantes señalan que Facebook es muy amigable, cómodo y de fácil acceso, características que permiten una mayor interactividad.

$\mathrm{Al}$ respecto las recomendaciones de los docentes, durante las entrevistas realizadas, consisten en crear una cuenta adicional con el perfil profesional con una imagen cálida pero exclusivamente destinada a fines académicos, de esta manera no se traspasaría el límite profesional. En este tema aún existe una amplia discusión sobre las fronteras entre lo profesional y lo social, o el rol del docente en tiempos de internet cuando hay una inmensa proliferación de información que podría convertirse en conocimiento.

El docente en la era virtual tiene el desafío de convertirse en un tutor y promotor del aprendizaje, pues los estudiantes ahora disponen de una variada gama de aplicaciones para aprender de acuerdo a sus intereses. Los nuevos tiempos exigen un nuevo perfil de docente, alguien más cercano a un facilitador, orientado a despertar el pensamiento crítico y la capacidad de problematizar la realidad y discriminar los datos.

Facebook sirve también para establecer grupos de acuerdo a las preferencias particulares, al respecto se encontró que un 79,50\% de estudiantes y un 57,20\% de profesores pertenecen a un grupo en Facebook con finalidades académicas. Esta realidad advierte que efectivamente Facebook se ha posicionado como un espacio 
que permite la interactividad con relación a tareas en el ámbito educativo. Tanto docentes como estudiantes pueden acceder a valiosa información de grupos de estudios locales o internacionales. A través de Facebook, conocen opciones de estudios, eventos académicos o participan en discusiones de grupos abiertos o cerrados referentes a sus preocupaciones profesionales.

Facebook se instaló entre docentes y estudiantes, de los resultados arrojaron que el 78,6\% de docentes y un $89,1 \%$ de estudiantes establecen que las redes sociales permiten intercambiar información y conocimiento de forma rápida y sencilla, así mismo un $61,4 \%$ de docentes y un $80,3 \%$ de estudiantes consideran que las redes sociales facilitan la innovación en los procesos de enseñanza-aprendizaje.

\section{ESTUDIANTES}

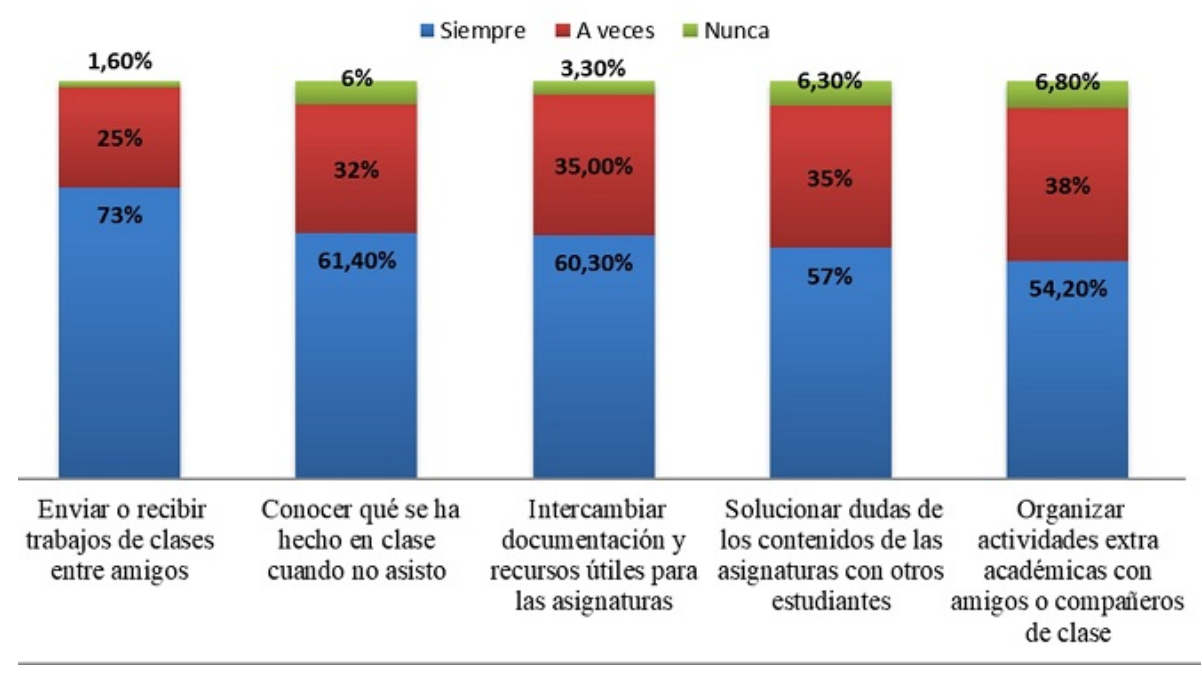

FIGURA 1.

Usos de Facebook realizados por los estudiantes de la Universidad de Cuenca durante el período 2016-2017 para actividades académicas Fuente: elaboración propia 


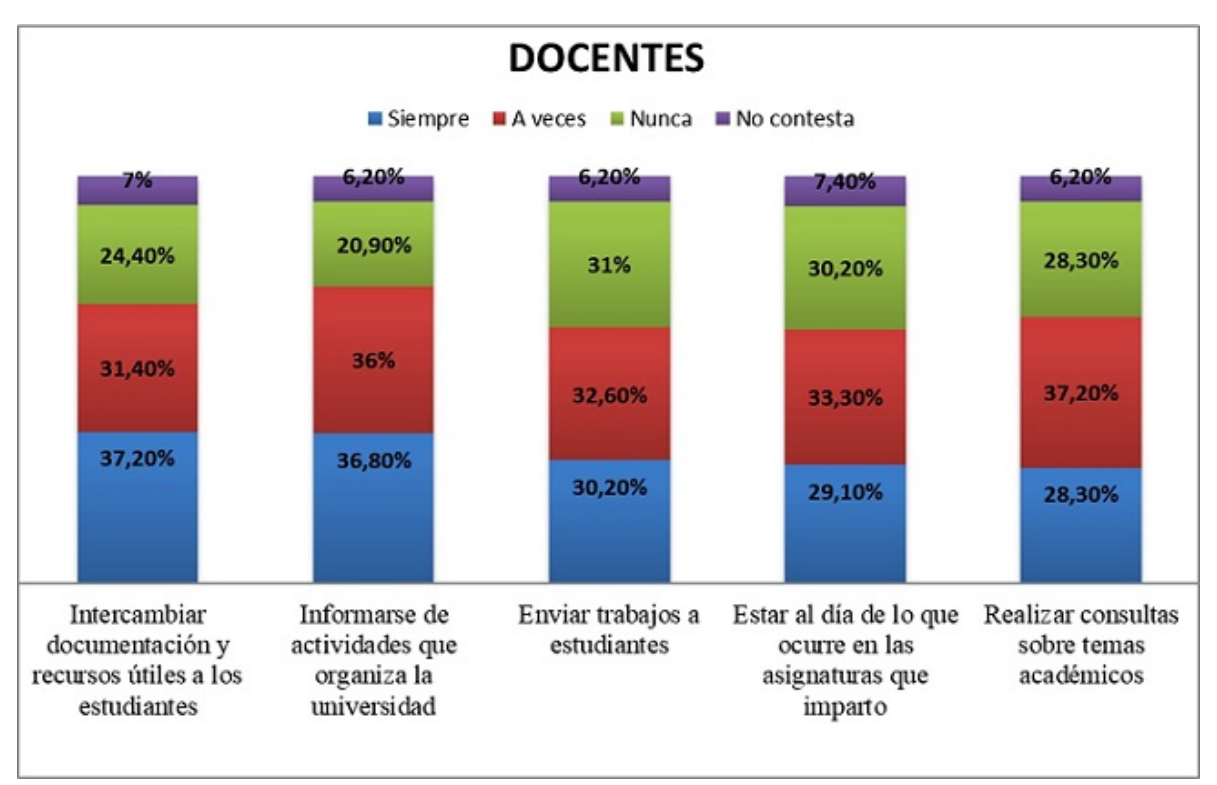

FIGURA 2.

Usos de Facebook para actividades académicas realizados por los docentes de la Universidad de Cuenca, período 2016-2017. Fuente: elaboración propia

De la investigación realizada (ver figuras 1 y 2), se desprende que los estudiantes utilizan el Facebook para actividades académicas, así un $73 \%$ siempre envía o recibe trabajos, un 60,3\% siempre intercambia información relacionada con las asignaturas, un $57 \%$ siempre soluciona dudas sobre las asignaturas y un $54,3 \%$ siempre organiza actividades académicas con sus compañeros de clase. Todas estas actividades las realizan independientemente de sus docentes, realidad que confirma la importancia de tomar en cuenta a esta plataforma para fomentar las tareas colaborativas virtuales. Además, se demuestran posibilidades cada vez mayores, de enlazar al menos cuatro elementos para fortalecer el proceso de aprendizaje: a) trabajo en el aula; b) trabajo colaborativo en red; c) trabajo autónomo; d) trabajo en equipo.

Como se puede ver en la figura 2, los docentes integran Facebook en las actividades académicas, así un $37,20 \%$ de docentes siempre utiliza esta red social para intercambiar documentación útil para los estudiantes; 30,20\% siempre envía trabajos a estudiantes a través de Facebook; 29,10\% siempre usa Facebook para estar al día con temas vinculados con las asignaturas y un $28,30 \%$ siempre realiza consultas sobre temas académicos. Es así como se evidencia el impacto que tiene Facebook como elemento complementario a las actividades de maestros y al mismo tiempo se advierte la importancia de considerar a esta red social como una herramienta de comunicación, socialización e intercambio de información con los estudiantes.

Esta investigación muestra las potencialidades de Facebook en el proceso educativo, pues al generar grupos de aprendizaje colaborativo permanente y diálogos horizontales se alienta el desarrollo de un pensamiento cuestionador y crítico sobre las temáticas abordadas. Los estudiantes se conectan a Facebook al menos 5 horas, esto podría ser aprovechado para una mejor interacción con relación a las problemáticas abordadas en las aulas de clase. 


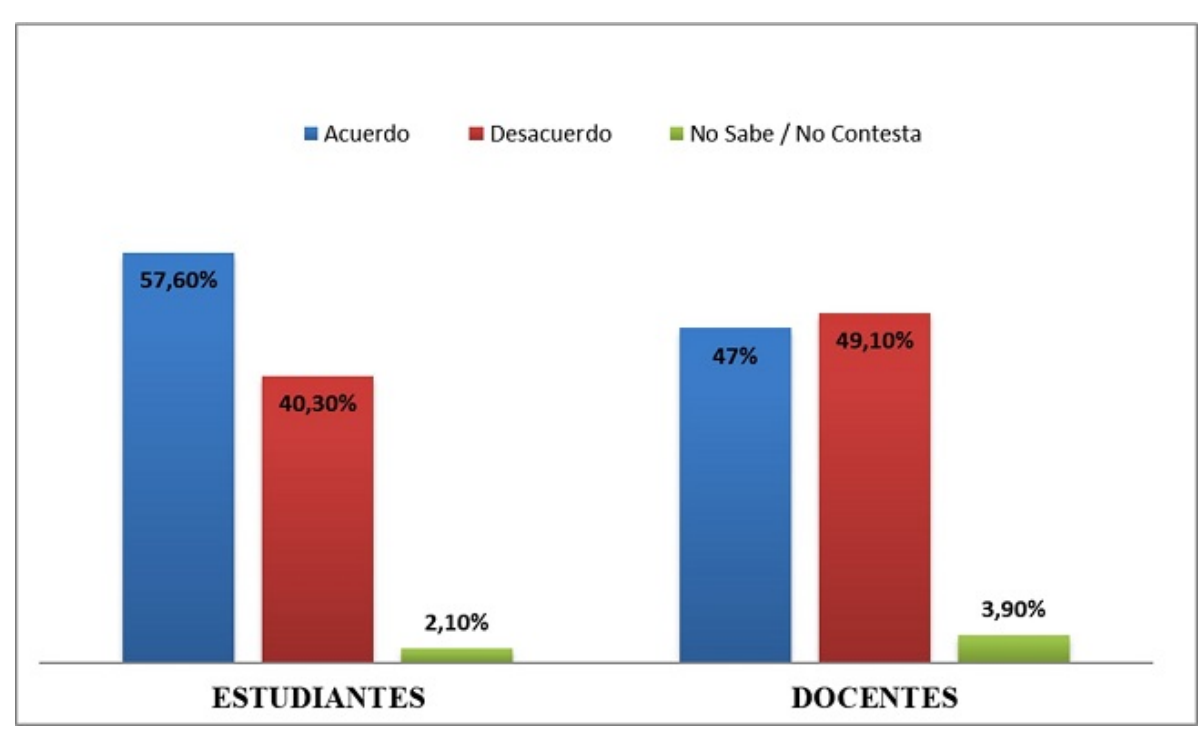

FIGURA 3.

Percepción del impacto negativo de las redes sociales en el rendimiento académico de los estudiantes universitarios

Fuente: elaboración propia

Con relación a los aspectos negativos (ver figura 3), un 57,6\% de estudiantes y un $47 \%$ de docentes señalan que las redes sociales tienen un impacto negativo en el rendimiento académico. En este tema, se reconoce que la cantidad de tiempo que los estudiantes están conectados a Facebook, puede interferir con las actividades estudiantiles. Para muchos profesores la presencia de internet ha provocado una suerte de escaneo plano de la realidad social, pues los estudiantes ya no profundizan un hecho sino tienen una mirada rápida y panorámica de los hechos, "los estudiantes no van más allá" (Ana Cecilia Salazar, docente Ciencias económicas, 19 octubre, 2017), su comportamiento en internet se traduce también en su realidad concreta, es decir están escaneando la realidad y no problematizando, por eso los docentes señalan la necesidad de "adaptar las metodologías a los nuevos tiempos recuperando una relación integradora entre el aprendizaje virtual y el aprendizaje vivencial" (Fredy Cabrera, docente Filosofía y Letras, 19 octubre, 2017). Al respecto se evidenció la necesidad de facilitar procesos de reflexión crítica y problematizadora de la realidad inmediata.

Se indagó también sobre las diferentes posiciones que tienen tanto docentes como estudiantes sobre las redes sociales, al respecto las respuestas son las siguientes: un $57,5 \%$ de docentes y un $66,4 \%$ de estudiantes consideran que las mismas constituyen una herramienta para democratizar el acceso a la información y la comunicación. Un $70,2 \%$ de docentes y un $75,2 \%$ de estudiantes señalan que estas plataformas son una herramienta de movilización y participación política.

De la investigación se desprende que los docentes ya no pueden estar ajenos a Facebook pues se ha convertido en un nuevo espacio público virtual en donde se exponen algunas de las preocupaciones de los jóvenes universitarios. Por eso mismo un acercamiento o inmersión en esta plataforma virtual permitirá que el docente conozca las formas de relacionamiento, las prácticas comunicativas y los temas que convocan a los estudiantes. Facebook es una herramienta útil de comunicación como soporte complementario para el aprendizaje pues brinda posibilidades de interactuar, establecer grupos colaborativos, tejer relaciones sociales en contextos de aprendizaje y generar proximidad franca y desprejuiciada entre docentes y estudiantes. 


\section{Conclusiones}

La investigación muestra claramente el surgimiento de un sujeto digital que aprende utilizando una pluralidad de recursos y estableciendo una relación natural con las redes virtuales, que deben ser aprovechadas también en procesos educativos. Tanto estudiantes como docentes están en Facebook, el cual es un espacio de acercamiento al "otro", pues permite conocerlo más allá del aula, saber de sus aficiones, los temas de interés, sus redes de amistades, grupos a los que pertenece y los entornos en los que interactúa. Sus discursos y prácticas están ahí, a la vista de todos. Este conocimiento del "otro", tiene una enorme potencialidad en el aprendizaje porque genera relaciones de proximidad que aportan para la democratización del proceso educativo, así mismo tanto docentes como estudiantes se encuentran en un escenario en donde son al mismo tiempo productores, receptores, distribuidores de contenidos en igualdad de condiciones. En este contexto, es importante considerar que el hecho educativo por parte del docente ya no se restringe a los muros universitarios ni se limita a sus actividades académicas, porque puede ocurrir en cualquier momento y en cualquier lugar (Marta-Lazo y Gabelas, 2017).

El rol del docente está cambiando frente a un sujeto digital que accede con facilidad a grandes bases de datos e información, visita tutoriales sobre los temas de las asignaturas, intercambia documentos, produce materiales educomunicativos como vídeos, genera relaciones de cooperación e interactividad en la red. En esta realidad, queda el enorme desafío de adaptar las redes sociales a los procesos educativos y potenciar una cultura crítica de uso inteligente y colaborativo de las plataformas digitales.

Se constata que este "nuevo espacio social" facilita una serie de interacciones en el ámbito educativo principalmente promueve relacionamientos entre grupos de pares, pues los estudiantes organizan para determinada asignatura un grupo en Facebook, que sirve para intercambiar información, compartir trabajos, estar actualizados con relación a las actividades y para contar con la asesoría de los docentes en temas puntuales. Esta realidad también nos muestra que los estudiantes están organizando sus actividades, de manera - muchas veces- independiente de sus profesores, en una nueva dinámica de interacciones o trabajo colaborativo surgido a partir de sus propios intereses.

De la indagación realizada son recientes las experiencias de docentes en la Universidad de Cuenca que han integrado el Facebook en su práctica pedagógica, sin embargo, se visualizan algunas potencialidades importantes. De las entrevistas se concluye que Facebook podría servir para compartir tareas de los estudiantes, estas no son solo analizadas por el docente, sino todos pueden aportar y comentar los trabajos de sus compañeros, esta realidad tiene aportes interesantes para el fortalecimiento de un entorno colaborativo, aprendizaje sobre el trabajo en equipo, generación de un sentimiento de identidad con la asignatura y la circulación de comentarios o debates en temas propuestos desde el docente y desde los estudiantes.

Los estudiantes universitarios usan Facebook no solamente para socializar, mostrarse o divertirse, sino para actividades académicas, de hecho, un $73 \%$ siempre comparten trabajos entre sus compañeros de clase y un $60,30 \%$ siempre intercambian información útil sobre las asignaturas. Esto muestra que las redes sociales constituyen una herramienta de comunicación para las actividades académicas.

Es muy notorio que Facebook podría convertirse en espacio virtual para mediar las relaciones entre profesores y estudiantes facilitando procesos de comunicación e interactividad, los cuales impulsarían un mejor entendimiento entre los dos sujetos del proceso educativo, considerando a la comunicación a través de las redes virtuales, como un soporte importante para la enseñanza-aprendizaje. De la investigación se desprende que tanto estudiantes como docentes, se van integrando como contactos recíprocos, situación que posibilitaría abrir nuevas dinámicas de conocimiento y comunicación mutua que redundan en las actividades académicas. Las redes virtuales se vislumbran como escenarios de interactividad docenteestudiante, estudiante-estudiante, para establecer diálogos fluidos de carácter horizontal aportando a la participación. Sin embargo, es preciso señalar que Facebook no es una plataforma pedagógica sino una 
herramienta complementaria que puede ser usada para mejorar la comunicación y el intercambio de información ( Tuñez y Sixto, 2012; Fondevila et al., 2015).

Se evidencia que los estudiantes conforman sus grupos en Facebook para compartir información relacionada con las asignaturas, esta situación podría proyectarse hacia la conformación de comunidades de aprendizaje, participación y colaboración entre los mismos estudiantes, en una dinámica cooperativa. En este escenario virtual con una presencia significativa de comunicaciones informales sobre la cotidianidad y sus historias personales, también brinda la oportunidad de recuperar el protagonismo para dejar de ser consumidores de información y conocimiento sino adentrarse en el territorio de la generación y producción de ese conocimiento, afirmación que nos obliga a un análisis permanente e interdisciplinario sobre el rol de estas redes virtuales en la comunicación y en la educación.

La hiperconectividad, permite desarrollar la experiencia vital en el tiempo real y el tiempo virtual, en el territorio inmediato, así como en el espacio público digital. Estas son algunas de las peculiaridades de un proceso en transición hacia la configuración de un sujeto digital que se enfrenta al desafío de superar el gregarismo por miedo a la soledad, para pensarse a sí mismo como sujeto con el mundo, como ser actuante a partir de una reflexividad con los otros.

En general los medios digitales denominados redes sociales podrían aportar a una mayor interacción humana, los estudiantes están usando estas herramientas, a diferencia de los docentes que aún tienen posiciones más tradicionales con relación a integrar estos elementos para mejorar la comunicación, para compartir o generar información relevante con relación a las asignaturas.

La realidad estudiada plantea que desde los docentes es necesario establecer procesos más abiertos para comprender las formas cómo los estudiantes se comunican, aprenden y conviven en estas dinámicas virtuales. No es posible estar fuera de esta comprensión, por el contrario, aprovechar las posibilidades que ofrece Facebook para integrar a los estudiantes en la creación de conocimientos.

Como señalan varios estudios de distintas universidades, las redes virtuales se podrían transformar en elementos integrados a las dinámicas educativas, pues actualmente ya no pueden ser ignoradas de la planificación docente (Spuny et al., 2011). Esta realidad muestra la urgencia de realizar ajustes metodológicos e innovaciones en las prácticas pedagógicas que permitan abordar estos aspectos desde una mirada cuestionadora que permita usar Facebook como elemento de comunicación (Fondevila et al., 2015). Al respecto de esta discusión, se propone consolidar un grupo académico interdisciplinario de debate, reflexión e investigación permanente sobre las redes virtuales como soporte de comunicación para la enseñanzaaprendizaje.

Las diferentes facultades están haciendo un uso parcial de Facebook para comunicar sobre aspectos referentes a la gestión institucional pues todavía no están muy claras, en las instancias formales, las estrategias comunicativas a partir de una mejor incorporación de las redes virtuales y su dimensión positiva para comunicar y generar interactividad.

Estamos viviendo el tránsito hacia la era digital, en este proceso es indispensable que esta transitividad sea desde el pensamiento crítico (Freire, 1969) para no caer en una suerte de transitividad ingenua que muchas veces es promovida desde las plataformas en internet, en este sentido cobra actualidad el pensamiento de Freire, pues las condiciones de imposición se mantienen.

En tiempos de hiperconectividad se hace preciso dejar la transitividad ingenua, que está presente en Facebook, caracterizada por un gregarismo forjado en el miedo a la soledad, la asunción de explicaciones mágicas o fabulosas a la realidad social, el culto a lo fácil e inmediato, la falta de argumentación y la sustitución del diálogo constructivo por la polémica; hacia una transitividad crítica, utilizando las redes sociales de internet para generar diálogos activos, responsabilidades sociales, receptando lo nuevo no como novelería sino desde la problematización y el conocimiento crítico. Asumiendo que estas plataformas están en nuestro presente y futuro, es necesario adaptarse críticamente a la nueva realidad. 


\section{Agradecimientos}

Al Departamento de Investigación de la Universidad de Cuenca (DIUC).

\section{Referencias}

Alcoceba Hernando, J. (2013). Juventud, tecnologías de la información y cambio social. Perspectivas y escenarios para la socialización y la participación. En F. Sierra (Coord.). Ciudadanía, tecnología y cultura. Nodos conceptualespara pensar la nueva mediación digital (pp. 181-210). GEDISA.

Alonso García, S., y Alonso García, M. (2014). Las redes sociales en las Universidades Españolas. Revista de Comunicación de la SEECI., XVI(33), 132-140. https://doi.org/10.15198/seeci.2014.33.132-140

Esquivel Gómez, I., y Rojas Kramer, C. (2014). Uso de facebook en ámbitos educativos universitarios. Apertura, 6(2). https://www.researchgate.net/publication/279533352_Uso_de_Facebook_en_ambitos_educativos_uni versitarios_Consideraciones_y_recomendaciones

Fondevila Gascón, J., Mir Bernal, P., Santana López Eva, Crespo, J., y Rom Rodríguez, J. (2015). La introducción de Facebook en el aula univesitaria en España: la percepción del estudiante. Revista Latinoamericana de Tecnología Educativa, 14(3), 63-73. https://doi.org/10.17398/1695-288X.14.3.63

Freire, P. (1969). La educación como práctica de la libertad. Siglo XXI.

Lambic, D. (2016). Correlation betwoon Facebook use for educational purposes and academic performance of studentes. Computers in Human Behavior, 61, 313-320. https://doi.org/10.1016/j.chb.2016.03.052

Marta-Lazo, C., \& Gabelas, J. (2017). Comunicación digital y educación. Teoría y práctica. Magisterio.

Martín Serrano, M. (2007). Teoría de la Comunicación. Madrid: McGraw Hill.

Martínez Martínez, J. (2017). Estudio de caso sobre la relación entre redes sociales y escuela en los procesos de aprendizaje. Universidad, Escuela y Sociedad, V(n), 46-62. Obtenido de https://www.researchgate.net/publication/321533166_Estudio_de_caso_sobre_la_relacion_entre_redes_s ociales_y_escuela_en_los_procesos_de_aprendizaje

Menéndez Echavarría, A., \& Sánchez Hernández, C. (2013). Uso de plataformas social media en la práctica docente universitaria. Signo y Pensamiento 32(63), 152-168. https://revistas.javeriana.edu.co/index.php/signoypensam iento/article/view/6948

Piscitelli, A. G. (2009). Facebook. Esa reiterada tensión entre la sobrepromesa y la invención de nuevos mundos. Revista de Universidad y Sociedad del Conocimiento, 6(1). https://www.redalyc.org/articulo.oa?id=78011179012

Piscitelli, A., Adaime, I., \& Binder, I. (2010). El Proyecto Facebook y la posuniversidad. Sistemas operativos sociales y entornos abiertos al aprendizaje. Ariel.

Rodríguez Gallego, M., López Martínez , A., \& Martín Herrera , I. (enero de 2017). Percepciones de los estudiantes de las Ciencias de la Educación sobre las redes sociales como metodología didáctica. (U. d. Sevilla, Ed.) Pixel Bit Revista de Medios y Educación, (50), 77-93. http://dx.doi.org/10.12795/pixelbit.2016.i50.05

Serrano, P. (2017). Comunicación Jibarizada. Editoria Científico-Técnica.

Spuny, C., González, J., Leixá, M., \& Gisbert, M. (2011). Actitudes y expectativas del uso educativo de las redes sociales en los estudiantes universitarios. Revista de Universidad y Sociedad del Conocimiento, 8(1), 171-185. https://ww w.raco.cat/index.php/RUSC/article/download/225630/306986

Sued, G. (2010). Pensando a Facebook, una aproximación colectiva por dimensiones. En P. Alejandro, I. Adaime, y I. Binder, (Comp.) El Proyecto Facebook y la post universidad: sistemas operativos, sociales y entornos abiertos al aprendizaje. (pp. 59-70). Ariel. de https://www.academia.edu/2125637/Pensando_a_Facebook_una_aproxim aci\%C3\%B3n_colectiva_por_dimensiones

Tuñez López, M., \& Sixto García , J. (2012). Las redes sociales como entorno docente, análisis del uso de facebook en la docencia universitaria. (U. d. Sevilla, Ed.) Pixel,Bit. Revista de Medios y Educación, (41), 77-92. https://www .redalyc.org/articulo.oa?id=36828247006 


\section{Notas}

* Artículo de investigación científica. Este artículo forma parte del Proyecto de investigación "Estudio sobre usos y aplicaciones de la red social Facebook en la Universidad de Cuenca (2015-2016)", ganador del XIV Concurso de Proyectos de Investigación del Departamento de Investigación de la Universidad de Cuenca.

\section{Licencia Creative Commons CC BY 4.0}

Cómo citar este artículo: Molina Loyola, C., Ortiz Vizuete, F., Ávila Solano, B., \& Maldonado Vélez, Á M. (2020). Facebook como herramienta de comunicación en procesos educativos. Signo y Pensamiento, 39(76). https://doi.org/10.11144/Javeriana.syp39-76.fhcp 